\author{
EDUCATION AND IMAGING
}

\title{
Hepatobiliary and Pancreatic: Giant hepatomegaly from hepatic hemagiomatosis
}

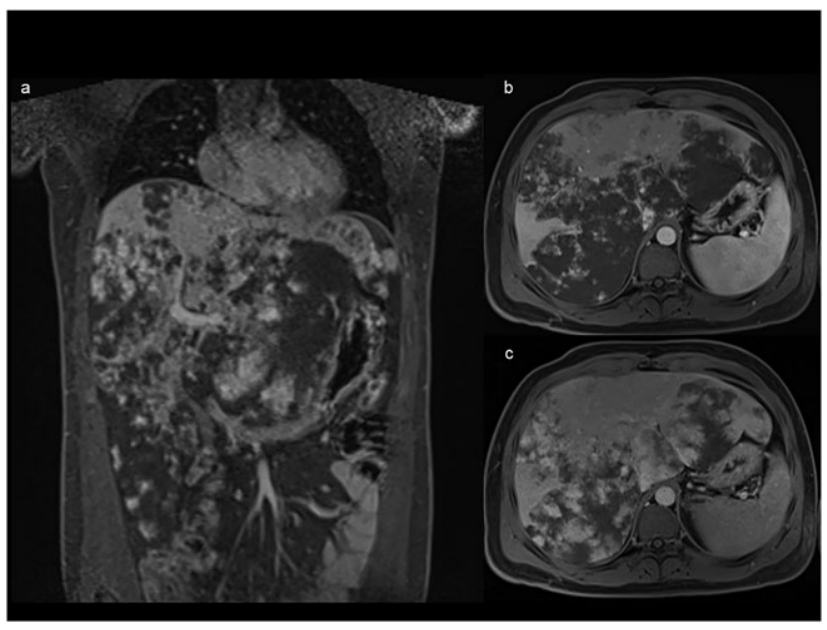

Figure 1 Abdominal magnetic resonance imaging showed an exuberant hepatomegaly (a) and countless hepatic confluent nodular lesions involving whole liver suggestive of hemangiomas (b and c).

A healthy 29-year-old man was presented with a 6-month history of abdominal discomfort. The patient had no personal or familiar prior history of relevant diseases or drug consumption.

Physical examination was unremarkable, except for a massive hepatomegaly. Blood test revealed only a slight elevation of $\gamma$-glutamil transpeptidase (111UI/L; normal range: 15-85UI/L). Abdominal computed tomography (CT) was the initial imaging assessment and showed multiple nodules throughout the liver parenchyma.

An abdominal magnetic resonance imaging with contrast, T2 and T1-weighed images, was then performed for further evaluation (Fig. 1). Abdominal magnetic resonance imaging depicted a giant hepatomegaly (cranial-caudal diameter: $26.7 \mathrm{~cm}$ ) (Fig.1a), countless hepatic confluent nodular lesions with high T2-weighted signal intensity and centripetal filling in post-contrast T1-weighted images (Fig. 1b,c) suggestive of hemangiomas. The hepatic lesions also caused extrinsic compression of the stomach, hepatic and portal veins.

Technetium-99 m-labelled Red Blood Cell scan (Tc99m RBC scan) and the fusion imaging of single-photon emission CT/CT

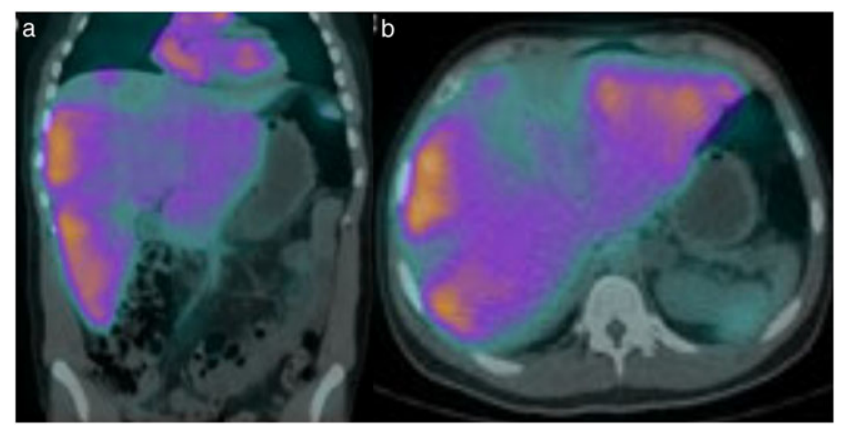

Figure 2 Single-photon emission computed tomography/computed tomography showed abnormal radioactive concentration in whole liver confirming diffuse hepatic hemangiomatosis ( $a$ and $b$ ).

(Fig. 2) confirmed hepatic hemangiomatosis and excluded extrahepatic hemangiomas.

Hepatic hemangiomatosis is characterized by diffuse replacement of hepatic parenchyma with hemangiomatous lesions, possibly associated with systemic hemangiomas. It is extremely rare in adults and usually seen in neonates.

Liver failure, consumptive coagulopathy, and high output heart failure are the most frequent symptoms, contributing to a poor clinical outcome.

Because of its general rareness and the few cases reported of long-term adult survival, the underlying etiology, clinical course and management remain unclear. Previous reports have suggested the role of estrogen and metoclopramide medications in the development diffuse hepatic hemangiomatosis. Although, this association is not yet confirmed.

After multidisciplinary discussion, our patient was referred for hepatic transplantation.

Contributed by

JM Costa (D*, D Costa ${ }^{*, \star,}$, F Vieira ${ }^{\dagger}$ and C Rolanda* ${ }^{*}, \S$ Departments of *Gastroenterology, ${ }^{\dagger}$ Radiology, Hospital de Braga, ${ }^{7}$ Life and Health Sciences Research Institute (ICVS), School of Health Sciences, University of Minho, and ${ }^{\S} I C V S / 3 B$ 's, PT Government Associate Laboratory, Guimarães/Braga, Portugal 